\title{
Will They Walk or Will They Talk? \\ Comparing Chinese and American Consumers
}

\author{
Anjala S. Krishen, Han-fen Hu, and Jordan Gunderson
}

\begin{abstract}
As the business world becomes less company driven and more customer-oriented, increasing loyalty becomes a critical factor to building a successful company (Zahay et al. 2012). Such loyalty is not easy to earn, but in the increasingly socially connected world, customers who are brand advocates serve as a key marketing tool (Sen and Lerman 2007). In effect, as a free resource that consumers can avail of at any time and from multiple sources, word of mouth (WOM) not only allows customers to tell their consumption stories and share their opinions, but it also allows them to research a seemingly independent view of a business (Moore 2012). As such, research abounds on the motivations driving WOM, whether positive or negative, such as self-enhancement, anger and retaliation, altruism, brand love, and many others (De Angelis et al. 2012).

Our chapter builds on existing literature by comparing the triggers for consumer satisfaction and WOM between Chinese and American consumers. We seek to further understand the drivers for positive or negative WOM in the two distinct cultures. We conduct an experiment using consumption type (hedonic vs. utilitarian) and service experience (positive versus negative) as our between-subject manipulations on 137 Chinese and 130 American consumers. Our findings indicate that consumers perceive higher level of satisfaction and switching cost, as well as provide higher WOM for positive than for negative service experiences. The relationship between service experience and switching cost is further moderated by consumption type and culture. Overall, our research offers an experimental view of consumers and how they react to negative and positive service experiences and hedonic versus utilitarian consumption types.
\end{abstract}

References available upon request.

\footnotetext{
A.S. Krishen $(\bowtie) \bullet$ H.-f. Hu • J. Gunderson

University of Nevada, Las Vegas, NV, USA

e-mail: anjala.krishen@unlv.edu; han-fen.hu@unlv.edu; jordangunderson3@gmail.com
} 